\title{
Alteração na cobertura florestal da região noroeste da Amazônia Matogrossense
}

\author{
Luani Rosa de Oliveira PIVA ${ }^{1 *}$, Rorai Pereira MARTINS NETO²
}

\author{
${ }^{1}$ Programa de Pós-Graduação em Engenharia Florestal, Universidade Federal do Paraná, Curitiba, PR, Brasil. \\ ${ }^{2}$ Programa de Pós-Graduação em Ciências Cartográficas, Universidade Estadual Paulista, Presidente Prudente, SP, Brasil. \\ *E-mail: luanipiva@yahoo.com.br
}

Recebido em outubro/2018; Aceito em abril/2019.

\begin{abstract}
RESUMO: Nos últimos anos, a intensificação das atividades antrópicas modificadoras da cobertura vegetal do solo em território brasileiro vem ocorrendo em larga escala. Para fins de monitoramento das alterações da cobertura florestal, as técnicas de Sensoriamento Remoto da vegetação são ferramentas imprescindíveis, principalmente em áreas extensas e de difícil acesso, como é o caso da Amazônia brasileira. Neste sentido, objetivou-se com este trabalho identificar as mudanças no uso e cobertura do solo no período de 20 anos nos municípios de Aripuanã e Rondolândia, Noroeste do Mato Grosso, visando quantificar as áreas efetivas que sofreram alterações. Para tal, foram utilizadas técnicas de classificação digital de imagens Landsat 5 TM e Landsat 8 OLI em três diferentes datas (1995, 2005 e 2015) e, posteriormente, realizada a detecção de mudanças para o uso e cobertura do solo. A classificação digital apresentou resultados excelentes, com índice Kappa acima de 0,80 para os mapas gerados, indicando ser uma ferramenta potencial para o uso e cobertura do solo. Os resultados denotaram uma conversão de áreas florestais principalmente para atividades antrópicas agrícolas, na ordem de $472 \mathrm{~km}^{2}$, o que representa uma perda de 1,3\% de superfície de floresta amazônica na região de estudo. Palavras-chave: conversão de áreas florestais; uso e cobertura do solo; classificação digital; análise multitemporal.
\end{abstract}

\section{Change in forest cover of the northwest region of Amazon in Mato Grosso state}

\begin{abstract}
In the past few years, the intensification of anthropic activities that modify the soil-vegetation cover in Brazil's land has been occurring on a large scale. To monitor the forest cover changes, the techniques of Remote Sensing of vegetation are essential tools, especially in large areas and with difficult access, as is the case of the Brazilian Amazon. The aim of this work was to identify the changes in land use and land cover, over the past 20 years, in the municipalities of Aripuanã and Rondolândia, Northwest of Mato Grosso State, in order to quantify the effective altered areas. Landsat $5 \mathrm{TM}$ and Landsat 8 OLI digital classification images techniques were used in three different dates (1995, 2005 and 2015) and, later, the detection to the land use and land cover changes. The digital classification showed excellent results, with kappa index above 0.80 for the generated maps, indicating the digital classification as a potential tool for land use and land cover. Results reflect the conversion of forest areas mainly for agricultural activities, in the order of $472 \mathrm{~km}^{2}$, representing a loss of $1.3 \%$ of Amazon forest surface in the study region.
\end{abstract}

Keywords: forest conversion; land use and land cover; digital classification; multitemporal analysis.

\section{INTRODUÇÃO}

No Brasil, o aumento expressivo da conversão de áreas de floresta para áreas de agropecuária vem ocorrendo em grande escala nos últimos anos, devido, principalmente, pelo aumento das demandas internas e externas por carne, soja e milho (MACEDO et al., 2012; NEPSTAD et al., 2014).

Entretanto, a intensificação dessas atividades buscando atingir demandas de mercado vem ocorrendo de maneira desenfreada, causando danos ao meio ambiente, como mudanças nas características físicas e químicas do solo (LONGO; ESPÍNDOLA, 2000; MAKEWITZ et al., 2004) na quantidade e qualidade da biomassa aérea (FEARNSIDE; BARBOSA, 1998), bem como o decorrente aumento das emissões de gases de efeito estufa (FEARNSIDE, 2002). Para comprovação da extensão e do impacto de tais danos, necessita-se da implementação de técnicas de monitoramento da vegetação e de alterações no uso do solo ao longo dos anos.

Devido à vasta extensão territorial e dificuldade de acesso às áreas, o trabalho de monitoramento das mudanças na ocupação do solo no Brasil, como é o caso do desmatamento ilegal, é uma atividade dispendiosa e onerosa (SOUZA JR. et al., 2003). Assim, o Sensoriamento Remoto é uma ferramenta muito útil para estudos de monitoramento da vegetação e uso do solo, pois a facilidade de acesso as imagens de satélites de média e baixa resolução espacial para análises multitemporais, bem como o aprimoramento das técnicas de interpretação, facilitam o mapeamento de mudanças temporais de pequena, média ou larga escala de tempo e/ou espaço (SCHAEFERSANTOS; LINGNAU, 2013).

No Bioma Amazônia, a utilização de técnicas de Sensoriamento Remoto é de grande importância, pois permite a obtenção de imagens mesmo em áreas de floresta densa (CÂMARA et al., 2015; RUFIN et al., 2015). Além disso, a partir das técnicas de detecção de mudanças, que corresponde à análise multitemporal de imagens adquiridas da mesma área geográfica, em diferentes períodos (dias, meses ou anos), é possível verificar possíveis alterações ocorridas em diferentes épocas (BOVOLO; BRUZZONE, 2005). 
O Estado do Mato Grosso ocupa a segunda posição no ranking de Estados com maiores índices de desmatamento na Amazônia Legal, ficando atrás apenas do Estado do Pará (AZEVEDO et al., 2016), sendo que a porção Norte do Mato Grosso está inserida dentro do chamado "arco do desmatamento" (VALERIANO et al., 2012). Além disso, o setor agropecuário engloba cerca de $21 \%$ do PIB do Estado (SEPLAN, 2014), acarretando no aumento de áreas desmatadas em prol dessa demanda econômica.

Nesse sentido, é de suma importância o conhecimento da dinâmica do uso e ocupação do solo ao longo dos anos, com objetivos de monitoramento, para que, então, órgãos ambientais fiscalizadores possam detectar, prevenir, mitigar e responsabilizar causadores de danos ambientais, como é o caso dos desmatamentos ilegais. Além disso, tais informações podem servir como ferramenta para o Poder Público na tomada de decisões, no que diz respeito à adoção de novas tecnologias e técnicas no setor agropecuário, visando tornar essa atividade menos impactante ao meio ambiente.

Neste contexto, o objetivo do trabalho foi identificar as mudanças do uso e cobertura do solo durante 20 anos nos municípios de Aripuanã e Rondolândia, região Noroeste do Estado do Mato Grosso.

\section{MATERIAL E MÉTODOS}

2.1. Área de Estudo

O estudo foi realizado nos municípios de Aripuanã e Rondolândia, região Noroeste do Estado do Mato Grosso, situando-se entre as latitudes $09^{\circ} 27^{\prime} \mathrm{S}$ e $10^{\circ} 51^{\prime} \mathrm{S}$ e longitudes $60^{\circ} 04^{\prime} \mathrm{O}$ e $60^{\circ} 49^{\prime} \mathrm{O}$ (Figura 1).

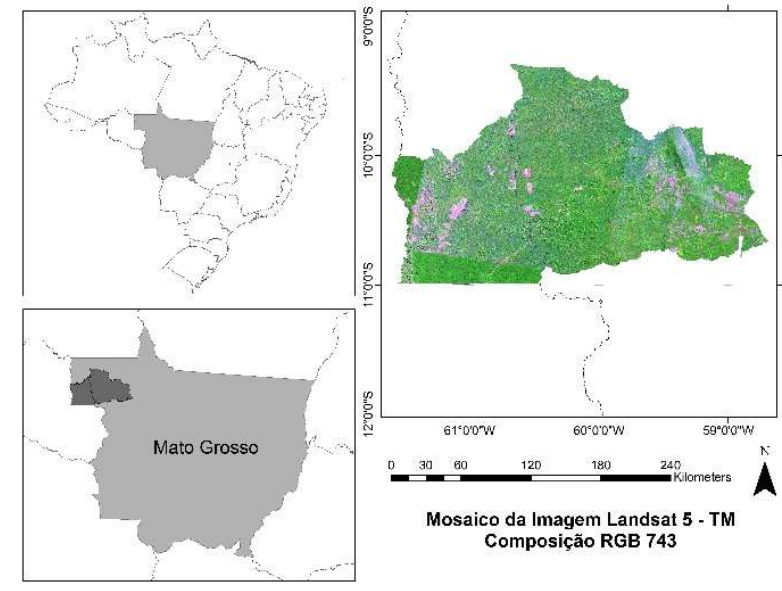

Figura 1. Localização da área de estudo nos municípios de Aripuanã e Rondolândia, Noroeste do Mato Grosso.

Figure 1. Location of the study area, covering the municipalities of Aripuanã and Rondolândia, Northwest of Mato Grosso State.

O clima da região é do tipo $\mathrm{Aw}$, de monções, com temperatura média anual de $26^{\circ} \mathrm{C}$ e precipitação anual de $2.200-2.500 \mathrm{~mm}$. O relevo da região é plano à suave ondulado, com altitude de 100 a 200 m (ALVARES et al., 2013).

Os solos predominantes da região são Argilossolo Vermelho-Amarelo distrófico e Latossolo Vermelho-Amarelo distrófico (IBGE, 2006). As características do relevo e dos tipos de solos na região favorecem o desenvolvimento de atividades de pecuária e cultivos agrícolas temporários, o que pode ser afirmado a partir de observação do mapa de aptidão agrícola do Estado, no qual a área de estudo está inserida na categoria "terras com aptidão regular para lavouras de ciclo curto e/ou longo" (SEPLAN, 2001).

A região de estudo está inserida dentro do chamado "arco do desmatamento", uma faixa territorial que abrange regiões dos Estados do Pará, Maranhão, Mato Grosso e Rondônia e que concentra as atividades de desmatamento na Amazônia brasileira (VALERIANO et al., 2012).

\subsection{Base de Dados}

Para a análise multitemporal das mudanças do uso e cobertura do solo ao longo de 20 anos, foram utilizadas imagens dos satélites Landsat 5 TM (Thematic Mapper) para os anos de 1995 e 2005 e do Landsat 8 OLI/TIRS (Operational Land Imager/Thermal Infrared Sensor) para o ano de 2015, as quais foram obtidas gratuitamente do catálogo de imagens do Serviço Geológico Norte-Americano (USGS), na plataforma Earth Explorer (Disponível em: https://earthexplorer.usgs.gov) (Tabela 1).

Devido à extensão territorial dos municípios, foram necessárias quatro imagens de satélite para recobrir toda a área de estudo (Tabela 1). Entre os anos, buscou-se imagens de uma mesma época do ano para evitar variações de iluminação, bem como diferentes estados fenológicos da vegetação. A época escolhida foi final do outono e início do inverno (meses de junho e julho), período de seca na região amazônica, com menor interferência de nuvens nas cenas.

Tabela 1. Dados de entrada das imagens dos satélites Landsat 5 TM para os anos de 1995 e 2005 e do Landsat 8 OLI/TIRS para o ano de 2015, nos municípios de Aripuanã e Rondolândia, MT.

Table 1. Input data from the satellites images of Landsat $5 \mathrm{TM}$ for the years of 1995 and 2005 and Landsat 8 OLI/TIRS for 2015, in the municipalities of Aripuanã and Rondolândia, Mato Grosso State.

\begin{tabular}{|c|c|c|c|c|}
\hline \multirow{2}{*}{$\begin{array}{l}\text { Especificações } \\
\text { Técnicas }\end{array}$} & \multicolumn{4}{|c|}{ Imagens 1995} \\
\hline & 1 & 2 & 3 & 4 \\
\hline Data & $22 / 07$ & $13 / 07$ & $13 / 07$ & $25 / 07$ \\
\hline Órbita/Ponto & $229 / 67$ & $230 / 67$ & $230 / 68$ & $231 / 67$ \\
\hline $\begin{array}{l}\text { Tempo Central } \\
\text { (GMT) }\end{array}$ & $13: 17: 20$ & $13: 23: 02$ & $13: 23: 26$ & $13: 28: 45$ \\
\hline Azimute do Sol $\left({ }^{\circ}\right)$ & 53,2360 & 51,5080 & 50,7728 & 50,2326 \\
\hline Elevação do Sol $\left({ }^{\circ}\right)$ & 39,32 & 38,4887 & 37,4099 & 38,0315 \\
\hline Especificações & \multicolumn{4}{|c|}{ Imagens 2005} \\
\hline Técnicas & 1 & 2 & 3 & 4 \\
\hline Data & $13 / 06$ & $04 / 06$ & $04 / 06$ & $11 / 06$ \\
\hline Órbita/Ponto & $229 / 67$ & $230 / 67$ & $230 / 68$ & $231 / 67$ \\
\hline $\begin{array}{l}\text { Tempo Central } \\
\text { (GMT) }\end{array}$ & $13: 50: 06$ & $13: 56: 13$ & $13: 56: 37$ & $14: 02: 27$ \\
\hline Azimute do Sol $\left({ }^{\circ}\right)$ & 42,424 & 42,807 & 42,034 & 42,456 \\
\hline Elevação do Sol $\left({ }^{\circ}\right)$ & 44,563 & 45,366 & 44,155 & 44,715 \\
\hline Especificações & \multicolumn{4}{|c|}{ Imagens 2015} \\
\hline Técnicas & 1 & 2 & 3 & 4 \\
\hline Data & $25 / 06$ & $02 / 07$ & $02 / 07$ & $23 / 06$ \\
\hline Órbita/Ponto & $229 / 67$ & $230 / 67$ & $230 / 68$ & $231 / 67$ \\
\hline $\begin{array}{l}\text { Tempo Central } \\
\text { (GMT) }\end{array}$ & 14:01:53 & 14:08:09 & 14:08:33 & $14: 14: 15$ \\
\hline Azimute do Sol $\left({ }^{\circ}\right)$ & 40,1044 & 40,7792 & 40,0330 & 39,9672 \\
\hline Elevacão do Sol $\left({ }^{\circ}\right)$ & 45,9777 & 46,0177 & 44,7799 & 46,0140 \\
\hline
\end{tabular}

\subsection{Processamento das imagens}

2.3.1 Mosaicagem e corregistro

A mosaicagem visa juntar um bloco de imagens sequenciais em uma única imagem. Esse processo foi realizado com o propósito de se obter uma imagem referente a toda área de estudo. Antes da mosaicagem, foram realizados ajustes de contrastes nos histogramas das imagens do bloco, com o intuito de minimizar as variações de brilho que pudessem comprometer a interpretação na etapa de 
classificação. Produzido o mosaico, esse foi recortado com base em um arquivo vetorial contendo o perímetro dos municípios, obtendo assim somente a parte da imagem que contém a área de interesse dos municípios.

$\mathrm{Na}$ aplicação da detecção de mudanças utilizando imagens multitemporais de uma mesma área, faz-se necessária a correspondência geográfica entres os pixels das imagens utilizadas. Assim, torna-se fundamental o corregistro entre as imagens, considerando uma dessas como referência (SCHAEFER-SANTOS; LINGNAU, 2013). O método de reamostragem utilizado na correção geométrica foi o Vizinho Mais Próximo, que mantem os valores originais de Números Digitais (ND) das imagens, com erro inferior a 0,5 pixel. As imagens foram sobrepostas para verificar possíveis discrepâncias de posicionamento, as quais não foram encontradas.

\subsubsection{Correção radiométrica e atmosférica}

O objetivo destas correções é de transformar os valores de NDs das imagens em valores físicos (refletância). De acordo com Ponzoni et al. (2015) esta etapa é necessária quando se faz análises comparativas de imagens tomadas por diferentes sensores ou análises multitemporais para compatibilizar a radiância registrada nas diferentes épocas, e suprimir as variações decorrentes do próprio sensor.

Primeiramente foi necessária a correção radiométrica que visa a transformação dos NDs em valores de radiância registrada pelo sistema do sensor. Para tal, foi utilizada a Equação 1 para cada banda dos satélites Landsat 5 e Landsat 8.

$\mathrm{L}_{\lambda}=\left(\frac{\mathrm{LMAX}_{\lambda}-\mathrm{LMI} \lambda}{\mathrm{ND}_{\max }-\mathrm{ND}_{\min }}\right)\left(\mathrm{ND}-\mathrm{ND}_{\min }\right)+\mathrm{LMIN}_{\lambda}$

em que: $L_{\lambda}$ é a radiância espectral registrada pelo sensor $\left(\mathrm{W} \mathrm{m}^{-2} \mathrm{sr}^{-1}\right.$ $\left.\mu \mathrm{m}^{-1}\right) ; L M A X_{\lambda}$ e $L M I N_{\lambda}$ são, respectivamente, a radiância espectral máxima e mínima que o sensor é capaz de registrar em determinado comprimento de onda $\left(\mathrm{W} \mathrm{m}^{-2} \mathrm{sr}^{-1} \mu \mathrm{m}^{-1}\right) ; N D$ é o valor do pixel a ser calibrado para radiância (adimensional); $N D_{\max }$ e $N D_{\min }$ são, respectivamente, os números digitais correspondentes ao $L M A X_{\lambda}$ e $L M I N_{\lambda}$ (adimensional).

Após a transformação dos NDs para radiância, foi realizada a correção dos efeitos atmosféricos para no fim obter-se nas imagens os valores de reflectância de superfície. Nesta etapa, foi utilizado o algoritmo FLAASH (Fast Line-ofSight Atmospheric Analysis of Spectral Hypercubes) que se baseia no modelo de transferência radiativa MODTRAN-4 (Moderate Resolution Atmospheric Radiance and Transmitance Model) para correção dos efeitos de espalhamento e absorção atmosférica (EXELIS, 2014). O modelo atmosférico utilizado foi o "Tropical" e o modelo de aerossol utilizado foi o "Rural".

2.4 Classificação digital

As amostras de treinamento foram selecionadas para classificação digital supervisionada das imagens, com o intuito de treinar o classificador. Essa é a primeira etapa de um processo de classificação digital e consiste na definição das características espectrais de cada classe temática estabelecida (PONZONI et al., 2015). As imagens Landsat foram classificadas de acordo com o método de Máxima Verossimilhança (Maximum-Likelihood).

Com base no Manual Técnico de Uso do Instituto Brasileiro de Geografia e Estatística (IBGE, 2006), as imagens foram classificadas de acordo com as seguintes categorias: áreas florestais; áreas expostas; áreas antrópicas agrícolas; e água (Tabela 2).

Tabela 2. Categorias estabelecidas para a classificação digital das imagens Landsat, com base na composição RGB 743 do satélite Landsat $5 \mathrm{TM}$.

Table 2. Categories established for the digital classification of Landsat images, based on the RGB 743 composition of the Landsat 5 TM satellite.

\begin{tabular}{ccc} 
Classe & Descrição & $\begin{array}{c}\text { Cor no } \\
\text { mapa } \\
\text { temático }\end{array}$ \\
\hline Áreas \\
florestais
\end{tabular}

A diferenciação das classes temáticas, bem como o cálculo de exatidão de cada classificação, foi baseada em amostras de treinamento de aproximadamente 10 pixels para as classes solo exposto, pastagem e floresta, e 12 pixels para a classe hidrografia. Um conjunto de amostras também foi selecionado para validação da classificação das imagens Landsat, cuja checagem foi feita com imagens do Google Earth ${ }^{\circledR}$. Após a classificação das imagens, foi aplicado um filtro de moda para eliminar pixels isolados.

Para avaliar o índice de acerto dos mapas temáticos, a partir de amostras de validação, matrizes de confusão para os 3 períodos de estudo foram geradas, indicando em sua diagonal principal a proporção dos pixels classificados corretamente e, na soma dos valores marginais das linhas e colunas, respectivamente, os erros de inclusão e omissão para cada classe temática (ROSENFIELD; FITZPATRICK-LINS, 1986).

A exatidão da classificação foi medida por meio do Coeficiente Kappa (COHEN, 1960), que utiliza todas as células da matriz de erro e não apenas os elementos da diagonal. Ou seja, mede a probabilidade de um pixel ser corretamente classificado em relação à probabilidade da classificação incorreta (DEMARCHI et al., 2011) (Equação 2).

$$
K=\frac{P_{0}-P_{e}}{1-P_{e}}
$$


em que: $P_{0}$ é a soma da coluna diagonal da matriz dividida pelo número total de amostras; $P_{e}$ é igual à $\sum x_{i+} x_{+i} /_{n^{2}}$, onde $x_{i+}$ é a soma da linha $i ; x_{+i}$ é a soma da coluna $i$ da matriz de confusão; $n$ é o número total de amostras.

A interpretação das classificações calculadas por meio do Coeficiente Kappa foi realizada pela associação de um intervalo de valores Kappa a um grau de qualidade do mapa temático (DEMARCHI et al., 2011), conforme metodologia desenvolvida por Landis; Koch (1977) (Tabela 3).

Tabela 3. Qualidade da classificação digital das imagens Landsat associada aos valores do Coeficiente Kappa.

Table 3. Classification quality of the Landsat images associated with values of the Kappa coefficient.

\begin{tabular}{cc}
\hline Valor do Coeficiente Kappa & Qualidade do mapa temático \\
\hline$<0,00$ & Péssima \\
$0,00-0,20$ & Ruim \\
$0,20-0,40$ & Razoável \\
$0,40-0,60$ & Boa \\
$0,60-0,80$ & Muito boa \\
$0,80-1,00$ & Excelente \\
\hline
\end{tabular}

2.5 Análise multitemporal e detecção de mudanças na cobertura vegetal

Após a classificação digital e cálculo da exatidão global das imagens Landsat, foi realizada uma análise multitemporal com o intuito de detectar a magnitude da mudança do uso e cobertura do solo na região dos municípios de Aripuanã e Rondolândia entre os anos de 1995 e 2015. Para isso, efetuou- se uma sobreposição dos mapas gerados para cada imagem para avaliar as alterações ocorridas durante todo o período. Por fim, a partir do mapa final, calculou-se o tamanho das áreas alteradas, em hectares, para cada uma das classes temáticas consideradas, com exceção da classe Água.

\section{RESULTADOS}

3.1. Classificação digital das imagens

A classificação digital das imagens indicou bom reconhecimento das classes temáticas após a comparação entre as imagens Landsat e imagens disponíveis no Google Earth ${ }^{\circledR}$, tendo em vista que poucos pontos de controle foram classificados erroneamente (Tabela 4), com acurácias globais, em média, superiores à $95 \%$. Além disso, a qualidade de todos os mapas temáticos foi considerada "Excelente", uma vez os Coeficientes Kappa calculados apresentaram valores superiores à 0,80. Para o ano de 1995, o Coeficiente Kappa calculado foi de 0,81 e acurácia global de $96,5 \%$, sendo que a maior confusão na classificação ocorreu para a classe temática "Áreas antrópicas agrícolas".

Para o ano de 2005, o Coeficiente Kappa foi igual à 0,86, com maior confusão na classe áreas florestais. A acurácia global da matriz foi de 96,5\% (Tabela 5). Na classificação para o ano de 2015, o Coeficiente Kappa foi superior aos observados nos outros anos, igual à 0,91 , demonstrando a excelente qualidade do mapa gerado. Além disso, a acurácia global foi de $97,6 \%$. A maior confusão na matriz foi observada para a classe áreas florestais.

Tabela 4. Matriz de confusão elaborada para avaliar o índice de acerto na classificação das imagens dos satélites Landsat 5 TM (anos de 1995 e 2005) e Landsat 8 OLI/TIRS (ano de 2015) para as quatro classes temáticas consideradas, nos municípios de Aripuanã e Rondolândia, MT. Table 5. Confusion matrix to evaluate the success rate in the image classification for the satellites Landsat 5 TM (years of 1995 and 2005) and Landsat 8 OLI/TIRS (year of 2015) for the four thematic classes, in the municipalities of Aripuanã and Rondolândia, Mato Grosso State.

\begin{tabular}{|c|c|c|c|c|c|c|}
\hline \multirow{7}{*}{ Real } & \multicolumn{6}{|c|}{ Mapa - Classificação 1995} \\
\hline & \multirow[b]{2}{*}{ Áreas florestais } & Áreas florestais & \multirow{2}{*}{$\begin{array}{c}\text { Áreas expostas } \\
-\end{array}$} & \multirow{2}{*}{$\begin{array}{c}\text { Áreas antr. } \\
2\end{array}$} & \multirow{2}{*}{$\begin{array}{c}\text { Água } \\
1\end{array}$} & \multirow{2}{*}{$\begin{array}{c}\text { Soma parcial } \\
154\end{array}$} \\
\hline & & 151 & & & & \\
\hline & Áreas expostas & - & 4 & 1 & - & 5 \\
\hline & Áreas antr. agrícolas & 2 & - & 7 & - & 9 \\
\hline & Água & - & - & - & 2 & 2 \\
\hline & Soma parcial & 153 & 4 & 10 & 3 & 170 \\
\hline \multirow{7}{*}{ Real } & \multicolumn{6}{|c|}{ Mapa - Classificação 2005} \\
\hline & \multirow{2}{*}{\multicolumn{3}{|c|}{$\begin{array}{c}\text { Áreas expostas } \\
-\end{array}$}} & \multirow{2}{*}{ Áreas antr. } & \multirow{2}{*}{$\begin{array}{c}\text { Água } \\
-\end{array}$} & \multirow{2}{*}{$\begin{array}{c}\text { Soma parcial } \\
144\end{array}$} \\
\hline & & & & & & \\
\hline & Áreas expostas & 2 & 9 & 1 & - & 12 \\
\hline & Áreas antr. agrícolas & 2 & 1 & 10 & - & 13 \\
\hline & Água & - & - & - & 1 & 1 \\
\hline & Soma parcial & 148 & 10 & 11 & 1 & 170 \\
\hline \multirow{7}{*}{ Real } & \multicolumn{6}{|c|}{ Mapa - Classificação 2015} \\
\hline & \multirow{4}{*}{$\begin{array}{c}\text { Áreas florestais } \\
\text { Áreas expostas } \\
\text { Áreas antr. agrícolas }\end{array}$} & Áreas florestais & \multirow{2}{*}{$\begin{array}{c}\text { Áreas expostas } \\
-\end{array}$} & \multirow{2}{*}{$\begin{array}{c}\text { Áreas antr. } \\
-\end{array}$} & \multirow{2}{*}{$\begin{array}{c}\text { Água } \\
-\end{array}$} & \multirow{2}{*}{$\begin{array}{c}\text { Soma parcial } \\
145\end{array}$} \\
\hline & & 145 & & & & \\
\hline & & - & 7 & - & - & 7 \\
\hline & & 2 & 1 & 12 & - & 15 \\
\hline & Água & - & - & 1 & 2 & 3 \\
\hline & Soma parcial & 147 & 8 & 13 & 2 & 170 \\
\hline
\end{tabular}

\subsection{Alteração da cobertura vegetal}

Para uma melhor avaliação do impacto das mudanças de uso do solo, os mapas de classificação para as 3 datas avaliadas foram comparados (Figura 2). Ao total, uma área de 37.788 $\mathrm{km}^{2}$ foi avaliada. As principais mudanças ao longo de 20 anos podem ser observadas na conversão de áreas florestais (verde) para áreas expostas (amarelo) e áreas antrópicas agrícolas (vermelho) entre os anos de 1995 (Figura 2.a) e 2005 (Figura 2b), em que 94.196 hectares de floresta foram suprimidos, além da conversão de áreas florestais e áreas expostas para áreas antrópicas agrícolas entre os anos de 2005 e 2015 (Figura 2c). 
Quanto a mudança ao longo dos 20 anos do uso do solo, houve uma expressiva alteração, principalmente no que se refere à cobertura vegetal. As áreas florestais sofreram redução na ordem de, aproximadamente, 47.214 hectares $\left(472 \mathrm{~km}^{2}\right)$, o que representa só para o município de Aripuanã, cerca de 11\% do valor de desmatamento acumulado até o ano de 2017 (INPE, 2017). A principal mudança ocorrida foi na classe de áreas antrópicas agrícolas, com aumento de $508 \mathrm{~km}^{2}$ ao longo de 20 anos. Além disso, o aumento das áreas expostas entre
1995 e 2005 foi expressivo, com aumento, ao total, de 69\%, o que corresponde a uma área de $745 \mathrm{~km}^{2}$.

Notou-se que de 2005 a 2015 houve uma redução de 780 $\mathrm{km}^{2}$ na área de solos expostos. Entretanto, essa mudança, apesar de ter representado aumento da cobertura florestal, também representou aumento expressivo de áreas antrópicas agrícolas, que aumentaram $311 \mathrm{~km}^{2}$ no período considerado (Figura 3).

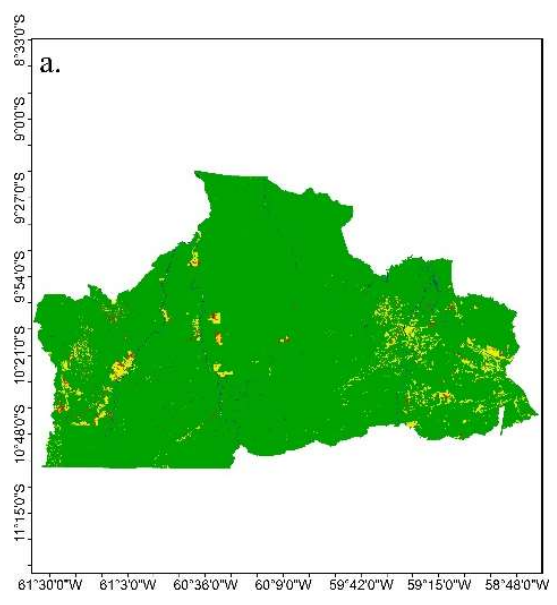

Árcas florestais $\quad \square$ Árcas cxpostas Árcas antrópicas agrícolas
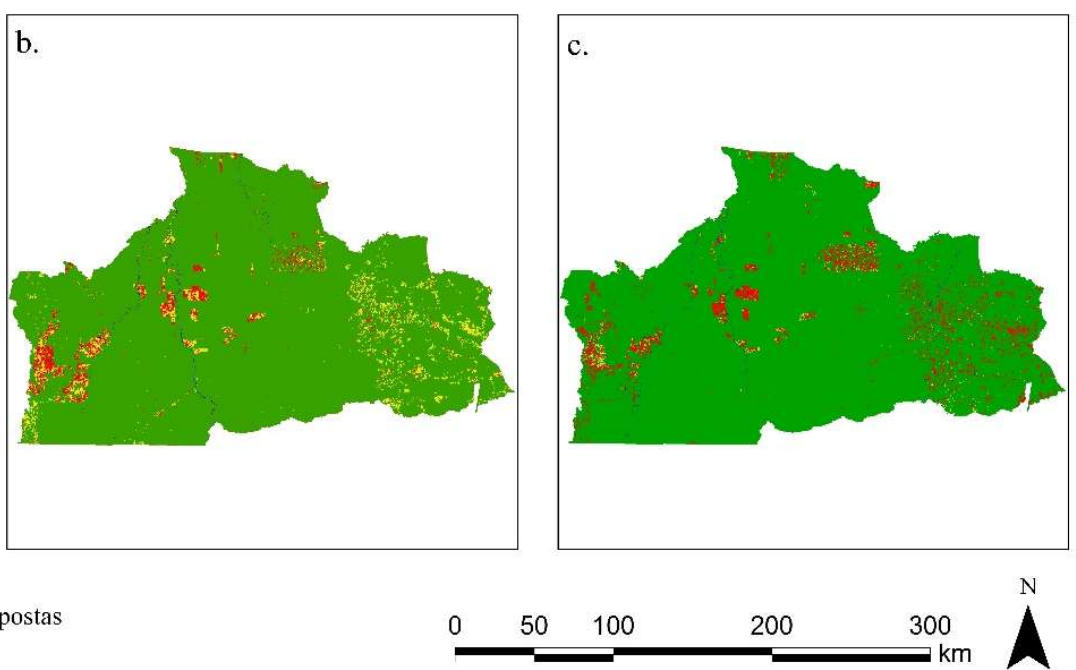

Figura 2. Alteração da cobertura vegetal nos municípios Aripuanã e Rondolândia, Estado do Mato Grosso: a. 1995 ; b. 2005 ; e c. 2015. Figure 2. Alteration of the vegetation cover in the municipalities of Aripuanã and Rondolândia, Mato Grosso State: a. 1995; b. 2005; and c. 2015 .

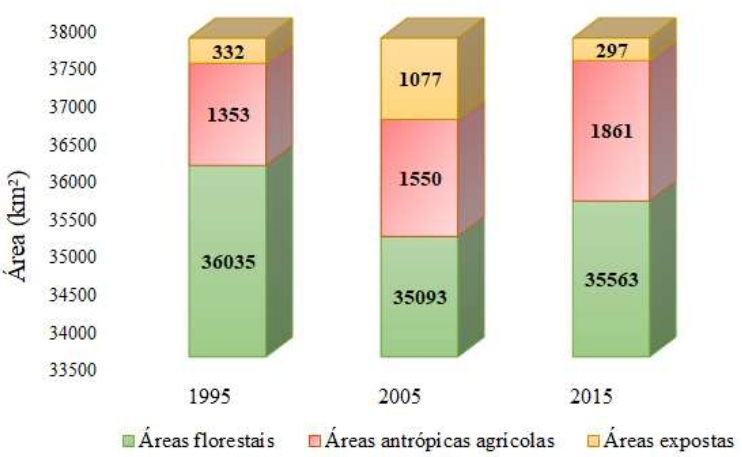

Figura 3. Comparação de áreas alteradas $\left(\mathrm{km}^{2}\right)$ para as classes áreas florestais, áreas antrópicas agrícolas e áreas expostas, nos anos de 1995, 2005 e 2015.

Figure 4. Comparison of altered areas $\left(\mathrm{km}^{2}\right)$ for the classes forest areas, agricultural anthropic areas and exposed areas, in the years 1995, 2005 and 2015.

\section{DISCUSSÃO}

A redução de áreas florestais nos últimos 20 anos ocorreu devido ao aumento das atividades agropecuárias na região, em decorrência das demandas internas por bens de consumo como grãos (soja e milho) e carne bovina (NEPSTAD et al., 2014). Além disso o crescimento das exportações de produtos agropecuários para outros países, com destaque para a China (ACIOLY et al., 2011), também contribuiu para a redução da cobertura vegetal na área estudada.

A região Noroeste do Mato Grosso é historicamente caracterizada como uma das principais fronteiras agropecuárias do Brasil (VIEIRA JUNIOR et al., 2014). Nos últimos 11 anos, houve um aumento nas áreas ocupadas por atividades agropecuárias no país na ordem de 16,5 milhões de hectares, sendo que, somente no Estado Mato Grosso, houve um aumento de 6,14 milhões de hectares (37\% do aumento observado no país) (IBGE, 2017).

Na região analisada, ao longo de 20 anos constatou-se uma redução de cerca de 46 mil hectares de área de florestas nativas (Figura 3), o que, considerando o Bioma Amazônia, representa prejuízos aos serviços ecológicos e ecossistêmicos que esta floresta proporciona.

Em estudo de Azevedo et al. (2016), constatou-se que, até o ano de 2015, houve um desmatamento acumulado de 4.029 $\mathrm{km}^{2}$ somente no município de Aripuanã - MT, o que equivale, aproximadamente, à 2,5 vezes o tamanho da cidade de São Paulo. Essa tendência para o estado do Mato Grosso foi corroborada por Rosan; Alcântara (2016), em estudo realizado no município de Claudia, no qual os autores demonstraram uma tendência da supressão de vegetação, natural ou regeneração, para fins econômicos relacionados à expansão da cultura da soja.

Quando ocorre a conversão de floresta para um outro tipo de uso, como por exemplo agropecuária, os impactos negativos dessas práticas acarretam em perdas de biodiversidade, degradação de florestas e degradação das áreas de bacias hidrográficas (FEARNSIDE, 2005).

Além do impacto ambiental, a condução inadequada das práticas agropecuárias é um entrave para o alcance de metas estabelecidas pelo país em acordos internacionais, a exemplo do Acordo de Paris (BRASIL, 2015), firmado pelo Brasil e mais 194 países. Nesse, foi estabelecido que, até 2030, o país deve reduzir em $43 \%$ as emissões de gases de efeito estufa, 
como o dióxido de carbono e, para alcançar essa meta, o governo brasileiro comprometeu-se a restaurar 12 milhões de hectares de floresta e atingir desmatamento ilegal zero na Amazônia brasileira.

Portanto, as técnicas de Sensoriamento Remoto, principalmente em regiões extensas e de difícil acesso, como é o caso da Amazônia brasileira, configuram-se como importantes ferramentas de monitoramento ambiental a serem utilizadas por órgãos fiscalizadores do governo municipal, estadual e federal. Dessa maneira, podem auxiliar no avanço das políticas de monitoramento do desmatamento, bem como no ordenamento do território visando práticas agropecuárias cada vez mais sustentáveis na região Noroeste do Estado do Mato Grosso.

\section{CONCLUSÕES}

Houve uma redução de 1,3\% $\left(472 \mathrm{~km}^{2}\right)$ das áreas de floresta nativa entre os anos de 1995 e 2015 , por conta de sua conversão para agropecuária, configurando-se como uma perda expressiva de área de floresta amazônica na região Noroeste do Mato Grosso ao longo de 20 anos;

A classificação digital supervisionada, aliada à análise multitemporal de detecção de mudanças, é eficaz para avaliação da magnitude das áreas de floresta convertidas para outros usos, como solo exposto e pastagem. Essas ferramentas também se mostraram precisas e de suma relevância para fins de monitoramento das mudanças no uso do solo e supressão da vegetação, para região inserida dentro do Bioma Amazônia.

\section{AGRADECIMENTOS}

Os autores agradecem à CAPES (Coordenação de aperfeiçoamento de pessoal de nível superior) e ao $\mathrm{CNPq}$ (Conselho Nacional de Desenvolvimento Científico e Tecnológico) pela concessão das bolsas de estudos que deram suporte à realização do presente trabalho.

\section{REFERÊNCIAS}

ACIOLY, L.; PINTO, E. C.; CINTRA, M. A. M.; CALIXTRE, A. B. As Relações bilaterais Brasil-China: a ascensão da China no sistema mundial e os desafios para o Brasil. Brasília: IPEA, 2011. 18 p. (Comunicados do Ipea, 85)

ALVARES, C. A.; STAPE, J. L.; SENTELHAS, P. C.; DE MORAES GONÇALVES, J. L.; SPAROVEK, G. Köppen's climate classification map for Brazil. Meteorologische Zeitschrift, Berlin, v. 22, n. 6, p. 711728, 2013. DOI: https://dx.doi.org/10.1127/09412948/2013/0507

AZEVEDO, A.; THUAULT, A.; SIMÕES, C.; CASTRO, I.; AMARAL, E. F. do; VALDIONES, A. P. Desmatamento na Amazônia-Mato-Grossense: análise das características do desmatamento e das políticas públicas implementadas. Ipam Amazônia; Instituto Centro Vida; PRODES, 2016. Disponível em: http://www.icv.org.br/wp-

content/uploads/2016/03/Desmatamento_Amazonia_pt.p df. Acesso em: 17 set 2018.

BOVOLO, F.; BRUZZONE, L. A detail-preserving scaledriven approach to change detection in multitemporal SAR images. IEEE Transactions on Geoscience and Remote Sensing, New York, v. 43, n. 12, p. 2963-2972, 2005.

BRASIL_REPÚBLICA FEDERATIVA DO BRASIL. Pretendida Contribuição Nacionalmente Determinada para consecução do objetivo da Convenção-Quadro das Nações Unidas sobre Mudança do Clima. Brasília, 2015. 10 p. Disponível em: $<$ http://www.itamaraty.gov.br/images/ed_desenvsust/BR ASIL-iNDC-portugues.pdf $>$. Acesso em: 09 set 2018.

CÂMARA, G.; SOTERRONI, A.; RAMOS, F.; CARVALHO, A.; ANDRADE, P.; SOUZA, R. S.; HAVLIK, P. Modelling Land Use Change in Brazil: 2000-2050 - A Report by the REED-PAC Project. São José dos Campos: INPE, 2015. 12 p. Disponível em: < http://pure.iiasa.ac.at/id/eprint/12115/7/lucbrazil_summar y.pdf $>$. Acesso em: 29 ago 2018.

COHEN, J. A coefficient of agreement for nominal scales. Educational and Psychological Measurement, Durham, v. 20, n. $1, \quad$ p. $37-46, \quad 1960$. DOI: https://dx.doi.org/10.1177/001316446002000104

DEMARCHI, J. C.; PIROLI, E. L.; ZIMBACK, C. R. L. Análise temporal do uso do solo e comparação entre os índices de vegetação NDVI e SAVI no município de Santa Cruz do Rio Pardo-SP usando imagens LANDSAT-5. Raega-O Espaço Geográfico em Análise, Curitiba, v. 21, p. 234-271, 2011. DOI: http://dx.doi.org/10.5380/raega.v21i0.17416

EXELIS_VISUAL INFORMATION SOLUTIONS EXCELISC. ENVIC. Fast Line-of-sight Atmospheric Analysis of Hypercubes (FLAASH). Boulder, USA. 2014.

FEARNSIDE, P. M.; BARBOSA, R. I. Soil carbon changes from conversion of forest to pasture in Brazilian Amazonia. Forest ecology and management, Amsterdam, v. 108, n. 1-2, p. 147-166, 1998. DOI: https://dx.doi.org/10.1016/S0378-1127(98)00222-9

FEARNSIDE, P. M. Time preference in global warming calculations: a proposal for a unified index. Ecological Economics, Amsterdam, v. 41, n. 1, p. 21-31, 2002. DOI: https://dx.doi.org/10.1016/S0921-8009(02)00004-6

FEARNSIDE, P. M. Desmatamento na Amazônia brasileira: história, índices e consequências. Megadiversidade, Belo Horizonte, v. 1, n. 1, p. 113-123, 2005.

IBGE INSTITUTO BRASILEIRO DE GEOGRAFIA E ESTATÍSTICA. Manual Técnico de Uso da Terra. 2 ed. 2006, $91 \mathrm{p}$.

IBGE INSTITUTO BRASILEIRO DE GEOGRAFIA E ESTATÍSTICA. Censo agropecuário 2017, 2017. Disponível em: < https://censos.ibge.gov.br/agro/2017/>. Acesso em: 12 set 2018.

INPE_INSTITUTO NACIONAL DE PESQUISAS ESPACIAIS. Projeto PRODES - dados por município, 2017, 2017. Disponível em: $<$ http://www.dpi.inpe.br/prodesdigital/prodesmunicipal.p hp>. Acesso em: 09 set 2018.

LANDIS, R.; KOCH, G. G. The measurement of observer agreement for categorical data. Biometrics, Washington, v. 33, n. 1, p. 159-174, 1977. DOI: https://dx.doi.org/10.2307/2529310

LONGO, R. M.; ESPÍNDOLA, C. R. Alterações em características químicas de solos da região Amazônica pela introdução de pastagens. Acta Amazonica, Manaus, v.30, n.1, p.71-80, 2000. DOI: http://dx.doi.org/10.1590/180943922000301080

MACEDO, M. N.; DEFRIES, R. S.; MORTON, D. C.; STICKLER, C. M.; GALFORD, G. L.; SHIMABUKURO, Y. E. Decoupling of deforestation and soy production in the southern Amazon during the late 2000s. Proceedings 
of the National Academy of Sciences, Allahabad, v. 109, n. 4 , p. 1341-1346, 2012. DOI: https://dx.doi.org/10.1073/pnas.1111374109

MAKEWITZ, D.; DAVIDSON, E.; MOUTINHO, P.; NEPSTAD, D. Nutrient loss and redistribution after forest clearing on a highly weathered soil in Amazonia. Ecological Applications, v. 14, n. sp4, p. 177-199, 2004. DOI: https://dx.doi.org/10.1890/01-6016

NEPSTAD, D.; MCGRATH, D.; STICKLER, C.; ALENCAR, A.; AZEVEDO, A.; SWETTE, B.; BEZERRA, T.; DIGIANO, M.; SHIMADA, J.; MOTTA, R. S. da; ARMIJO, E.; CASTELLO, L.; BRANDO, P.; HANSEN, M. C.; MCGRATH-HORN, M.; CARVALHO, O.; HESS, L. Slowing Amazon deforestation through public policy and interventions in beef and soy supply chains. Science, Washington, v. 344, n. 6188, p. 1118 1123 , 2014.

DOI: https://dx.doi.org/10.1126/science. 1248525

PONZONI, F. J.; SHIMABUKURO, Y. E.; KUPLICH, T. M. Sensoriamento remoto da vegetação. 2. ed. rev. e aum. São Paulo: Oficina de Textos, 2015. 176 p.

ROSAN, T. M.; ALCÂNTARA, E. Detecção de mudanças de uso e cobertura da terra na amazônia legal matogrossense: o estudo de caso do município de Cláudia (MT). Revista Brasileira de Cartografia, Uberlândia, v. 68, n. 5, 2016.

ROSENFIELD, G. H.; FITZPATRICK-LINS, K. A coefficient of agreement as a measure of thematic classification accuracy. Photogrammetric Engineering and Remote Sensing, Falls Church, v. 52, n. 2, p. 223227, 1986.

RUFIN, P.; MÜLLER, H.; PFLUGMACHER, D.; HOSTERT, $P$. Land use intensity trajectories on Amazonian pastures derived from Landsat time series. International Journal of Applied Earth Observation and Geoinformation, Enschede, v. 41, p. 1-10, 2015. DOI: https://dx.doi.org/10.1016/j.jag.2015.04.010
SCHAEFER-SANTOS, J. S.; LINGNAU, C. Monitoramento em área de catástrofe ambiental através de técnica de detecção de mudanças. Cerne, Lavras, v. 19, n. 1, p. 133140, 2013. DOI: http://dx.doi.org/10.1590/S010477602013000100016

SEPLAN SECRETARIA DE ESTADO DE PLANEJAMENTO E GESTÃO. Mapa de solos do Estado de Mato Grosso. Cuiabá: Secretaria de Planejamento, 2001. Disponível em: $<$ http://www.seplan.mt.gov.br/-/3952013-dsee-mapas1.500.000? ciclo $=\mathrm{cv}$ gestao inf $>$. Acesso em: 17 set 2018 . SEPLAN SECRETARIA DE ESTADO DE PLANEJAMENTO E GESTÃO. Mato Grosso - Produto Interno Bruto 2014. Cuiabá: Secretaria de Planejamento, 2014. Disponível em: <http://www.seplan.mt.gov.br//produto-interno-bruto? ciclo $=\mathrm{cv} \_g e s t a o \_i n f>$. Acesso em: 30 ago 2018.

SOUZA JR., C.; FIRESTONE, L; SILVA, M. L.; ROBERTS, D. A. Mapping forest degradation in the Eastern Amazon from SPOT 4 through spectral mixture models. Remote Sensing of Environment, New York, v. 87, n. 4, p. 494506, 2003.2 DOI: https://dx.doi.org/10.1016/j.rse.2002.08.002

VALERIANO, D. M.; ESCADA, M. I. S.; CÂMARA, G.; KAMPEL, S. A.; MAURANO, L. E. P.; DE ALMEIDA, C. A.; MONTEIRO, A. M. V. Dimensões do desmatamento na Amazônia Brasileira. In: MARTINE, G. (Ed.). População e sustentabilidade na era das mudanças ambientais globais: contribuições para uma agenda brasileira. Belo Horizonte: ABEP, 2012. p. 223238.

VIEIRA JUNIOR, P. A.; FIGUEIREDO, E. V. C.; REIS, J. C. Alcance e limites da agricultura para o desenvolvimento regional: o caso de Mato Grosso. In: BUAINAIN, A. M.; ALVES, E.; SILVEIRA, J. M. da; NAVARRO, Z. (Ed.). O mundo rural no Brasil do século 21: a formação de um novo padrão agrário e agrícola. Brasília, DF: Embrapa, 2014. p. 1125-1156. 